\title{
One Repetition Maximum
}

National Cancer Institute

\section{Source}

National Cancer Institute. One Repetition Maximum. NCI Thesaurus. Code C122034.

The maximum amount of force that can be generated in one maximal muscle contraction or the maximum amount of weight that can be cycled through one repetition of a particular movement or exercise. 